Accretion Phenomena and Related Outfiows, IAU Colloquium 163

ASP Conference Series, Vol. 121, 1997

D.T. Wickramasinghe, L. Ferrario, and G.V. Bicknell, eds.

\title{
The Nature and Evolutionary Status of GRO J1744-28
}

\author{
P. C. Joss and S. Rappaport
}

Massachusetts Institute of Technology

\begin{abstract}
GRO J1744-28 is the first known X-ray source to display both bursts and periodic pulsations. This source may thus provide crucial clues that will lead to an understanding of the differences in the nature of the X-ray variability among accreting neutron stars. We deduce that the magnetic field of the neutron star is relatively weak $\left(\sim 8 \times 10^{10} \mathrm{G}\right)$ but, nevertheless, sufficiently strong to funnel the accretion flow onto the magnetic polar caps and suppress the thermonuclear flashes that would otherwise give rise to type I X-ray bursts. We also present a series of interrelated arguments which demonstrate that the observed bursts are of type II and probably result from an instability associated with the interaction of the neutron-star magnetic field with the inner edge of the accretion disk. From these results, we infer that X-ray pulsars, GRO J1744-28, the Rapid Burster, and the type I X-ray bursters may form a sequence of possible behaviors among accreting neutron stars, with the strength of the magnetic field serving as the crucial parameter that determines the mode of X-ray variability. The companion star in the GRO J1744-28 binary system is probably a very low-mass $\left(\sim 0.2 M_{\odot}\right)$ giant that is in the final stages of losing its hydrogen-rich envelope. We have carried out binary evolution calculations which show that (1) if the mass of the giant was $\sim 1 M_{\odot}$ when mass transfer onto the neutron star commenced, then the orbital period and the core mass of the giant have increased from $\sim 1$ to $\sim 11.8$ days and from $\sim 0.15$ to $\sim 0.21 M_{\odot}$, respectively, during the mass-transfer epoch, which has lasted for $\sim 8 \times 10^{8} \mathrm{yr},(2)$ the present long-term average X-ray luminosity is $\sim 4 \times 10^{36} \mathrm{ergs} \mathrm{s}^{-1}$, which is at least two orders of magnitude lower than the luminosity at the peak of the transient outburst, and (3) the predicted long-term equilibrium rotation rate of the neutron star is remarkably close to the observed pulse rate. The transient nature of GRO J1744-28 may well be related to the final stages of dissipation of the envelope of the giant companion.
\end{abstract}

\section{Introduction}

The hard X-ray transient GRO J1744-28, discovered by Fishman et al. (1995) in 1995 December, is the first known X-ray source to display both bursts and periodic pulsations. The $\sim 0.5 \mathrm{~s}$ pulsations, whose existence demonstrates that the underlying object is an accreting neutron star with a magnetic field sufficiently strong to funnel the accretion flow onto its magnetic polar caps, have been found to be present in both the burst emission (Kouveliotou et al. 1996a) 
and the persistent emission between bursts (Finger et al. 1996a). This source may thus prove to be a Rosetta stone that will link our understanding of the $\mathrm{X}$-ray pulsars and the X-ray bursters. The spectra of the bursts and persistent emission are similar (Briggs et al. 1996; Swank 1996), with a characteristic photon energy of $\sim 14 \mathrm{keV}$, which is comparable to most other X-ray pulsars but higher than other $\mathrm{X}$-ray bursters. The persistent emission also displays quasi-periodic oscillations (QPOs) (Zhang et al. 1996), which are also likely to result from the presence of a weak but non-negligible neutron star magnetic field. Early in the outburst, the pulse period was found to be decreasing at a rate of $\sim 6 \times 10^{-5} \mathrm{~s} \mathrm{yr}^{-1}$ (Finger et al. 1996b); according to the theory of accretion torques in X-ray pulsars, this spinup rate corresponds to a luminosity of $\sim 5 \times 10^{38} \mathrm{ergs} \mathrm{s}^{-1}$. Combined with the upper limit to the persistent X-ray flux of $\sim 2 \times 10^{-7} \mathrm{ergs} \mathrm{cm} \mathrm{cm}^{-2} \mathrm{~s}^{-1}$ that was observed at about the same time (Fishman et al. 1996; Swank 1996), this implies a source distance of $\gtrsim 5 \mathrm{kpc}$; the relatively large distance is consistent with the proximity of the source to the direction of the galactic center. The detection of Doppler variations in the pulse period (Finger et al. 1996b) reveals that the neutron star is a member of a binary system, with an orbital period of 11.8 days and with a mass function $\left(1.31 \times 10^{-4} M_{\odot}\right)$ that is one of the smallest among all known binaries. The very small mass function implies either that we are viewing the system almost exactly pole-on, or that the companion star is of very low mass $\left(\lesssim 0.2 M_{\odot}\right)$.

We here present a model for the source and the binary system that is consistent with all of the observational facts. In our model, which is based in part on detailed binary evolution calculations, (i) the bursts are of type II (previously observed only in the Rapid Burster, MXB1730-335 [Lewin et al. 1976]) and are the result of an instability in the accretion flow onto the weakly magnetized neutron star, (ii) the companion is an evolved star that is driving mass transfer through its internal evolution and that has lost nearly its entire hydrogen-rich envelope through Roche-lobe overflow, and (iii) the transient nature of the source may well be related to the final stages of the dissipation of the companion's envelope.

\section{Nature of the X-ray bursts}

The observed behavior of GRO J1744-28 differs from that of type I X-ray burst sources, and from the predictions of the widely accepted theory that such bursts result from thermonuclear flashes in the surface layers of an accreting neutron star, in at least six important ways. First, the rate of bursting in GRO J1744-28 was observed to be between $\sim 1.6$ and $\sim 18$ per hour at various times during the transient outburst (Fishman et al. 1995; Kommers \& Fox 1996; Swank 1996). These rates are substantially higher than both the sustained rates of bursting observed in any type I X-ray burst source and the bursting rates predicted by the thermonuclear flash model (unless the bursts are very weak; see Joss \& Rappaport [1984] and Lewin et al. [1993] for reviews and references). Second, when the source was first detected the ratio, $\alpha$, of persistent X-ray luminosity to time-averaged burst luminosity was no higher than $\sim 4$ (Lewin et al. 1996). This is in contrast with the much higher sustained $\alpha$-values of all known type I burst sources (Lewin \& Joss 1981; Joss \& Rappaport 1984) and is also in disagreement with the predictions of the thermonuclear flash model 
(Joss 1977; Lewin \& Joss 1981; Joss \& Rappaport 1984). Third, the essentially identical mean photon energies of the bursts and the persistent emission suggest that the size of the X-ray emitting region decreases during burst decline. This behavior is similar to that of the type II bursts from the Rapid Burster but differs from that of type I X-ray bursts, wherein the size of the emitting region is about constant during relatively weak bursts and expands near the peaks of many of the strongest bursts (Lewin \& Joss 1981; Joss \& Rappaport 1984). Fourth, the presence of periodic pulsations in both the persistent emission and the burst emission indicates that the underlying object is a neutron star with a magnetic field that is sufficiently strong to funnel the accretion flow onto the magnetic polar caps. Numerical calculations (Joss \& Li 1980; see also Bildsten \& Brown 1996) of the behavior of the surface layers of an accreting neutron star indicate that such funneling should strongly tend to suppress thermonuclear flashes. Fifth, as argued below, the time-averaged persistent luminosity of this source during the peak of the transient episode is likely to have been quite high, comparable to or even slightly greater than the Eddington limit for spherical accretion onto an unmagnetized object. Again, theoretical calculations (Joss 1978; Ayasli \& Joss 1982) indicate that thermonuclear flashes will be strongly suppressed at the high accretion rates necessary to generate such luminosities. Sixth, Giles \& Strohmayer (1996) have discovered a large number of very weak bursts from GRO J1744-28, with complex patterns in the recurrence intervals and amplitudes of the bursts. These patterns are remarkably similar to the patterns in the type II bursts observed in the Rapid Burster.

The behavior of the QPOs observed in GRO J1744-28 is anomalous, compared to that observed in many other X-ray sources thought to be accreting, weakly magnetized neutron stars (Alpar \& Shaham 1985), in that the peak frequency of the QPOs is roughly independent of the source intensity (Morgan 1996). (It is interesting, in this regard, that the Rapid Burster also displays QPOs [Tawara et al. 1982] whose behavior does not fit into the standard categories for QPO sources; see Lewin et al. [1996] for a discussion and additional references.) The anomalous behavior notwithstanding, we shall assume that the peak QPO frequency is approximately equal to the Keplerian rotation frequency, $\nu_{\mathrm{k}}$, at the inner edge of the accretion disk (see, e.g., Alpar \& Shaham 1985). The strongest peak in the power spectrum of the QPOs lies at $\sim 40 \mathrm{~Hz}$, but peaks are also discernible at $\sim 20$ and $\sim 60 \mathrm{~Hz}$ (Zhang et al. 1996). We shall assume, therefore, that the fundamental frequency is at $20 \mathrm{~Hz}$ and that the 40 and $60 \mathrm{~Hz}$ peaks are higher harmonics, probably resulting from the existence of two accreting polar caps on the neutron star.

If we neglect unknown correction factors of order unity, we find that the radius of the neutron-star magnetosphere is given by

$$
r_{\mathrm{m}} \simeq 1.6 \times 10^{8} \mu_{30}^{4 / 7} L_{38}^{-2 / 7} \mathrm{~cm}
$$

(Rappaport \& Joss 1977), where $\mu_{30}$ is the neutron-star magnetic moment (for an assumed dipole field) in units of $10^{30} \mathrm{G} \mathrm{cm}^{3}$ and $L_{38}$ is the accretion-driven luminosity in units of $10^{38} \mathrm{ergs} \mathrm{s}^{-1}$. If we take $r_{\mathrm{m}}$ to be the radius of the inner edge of the accretion disk, then

$$
\nu_{\mathrm{k}} \simeq 1.1 B_{12}^{-6 / 7} L_{38}^{3 / 7} \mathrm{~Hz}
$$


where $B_{12}$ is the magnetic field strength at the magnetic polar caps of the neutron star, in units of $10^{12} \mathrm{G}$. Moreover, the theory of accretion torques in $\mathrm{X}$-ray pulsars (Rappaport \& Joss 1977), when combined with the observed pulse period and spinup rate of GRO J1744-28 and a canonical neutron-star mass of $1.4 M_{\odot}$ (Joss \& Rappaport 1976) and radius of $10 \mathrm{~km}$, gives an accretion rate of $\sim 2 \times 10^{-8} B_{12}^{-1 / 3} M_{\odot} \mathrm{yr}^{-1}$ and a luminosity of ${ }^{1}$

$$
L \simeq 2 \times 10^{38} B_{12}^{-1 / 3} \mathrm{ergs} \mathrm{s}^{-1} .
$$

Combining Equations (2) and (3) and taking $\nu_{k}$ to be $\sim 20 \mathrm{~Hz}$, we obtain

$$
B \simeq 8 \times 10^{10} \mathrm{G} \text { and } L \simeq 5 \times 10^{38} \mathrm{ergs} \mathrm{s}^{-1} .
$$

We emphasize that if the current pulse period is close to the long-term equilibrium rotation period of the neutron star, then the results of the binary evolution calculations presented in $\S 3$ below provide independent confirmation that the magnetic field strength is close to that given in Equation (4), irrespective of our association of $\nu_{\mathrm{k}}$ with the fundamental frequency of the QPOs.

The luminosity given in Equation (4) is comparable to that of many other $\mathrm{X}$-ray pulsars but is substantially higher than that of any known type I X-ray burster, all of which have luminosities of $\lesssim 10^{37} \mathrm{ergs} \mathrm{s}^{-1}$ (Lewin \& Joss 1981; Joss \& Rappaport 1984; Lewin et al. 1993). In fact, as noted above, this luminosity is inconsistent with the conditions required by the thermonuclear flash model for the production of type I bursts. If the source emission is roughly isotropic, then this luminosity, combined with the persistent X-ray emission observed near the peak of the transient outburst (Fishman et al. 1996; Swank 1996), implies a source distance, $d$, of $\sim 5 \mathrm{kpc}$. In light of the possibility that the persistent emission was somewhat overestimated due to source confusion in the vicinity of the galactic center (Paciesas et al. 1996), as well as the uncertainties inherent in our estimate of $L$, this estimate of $d$ is consistent with a source distance comparable to that of the galactic center $(\sim 8 \mathrm{kpc})$. If, instead, the luminosity were compatible with that expected for a type I burster, then GRO J1744-28 would be a foreground object with a distance $\lesssim 1 \mathrm{kpc}$, and the directional agreement with the galactic center would have to be a coincidence.

The magnetic field strength given in Equation (4) is substantially lower than the field strengths ( $\sim 10^{12} \mathrm{G}$; see Joss \& Rappaport [1984] for references) that have been inferred for other X-ray pulsars. It thus seems that a field strength of $\sim 8 \times 10^{10} \mathrm{G}$ is sufficient to funnel the accretion flow onto the magnetic polar caps of an accreting neutron star. The nearly sinusoidal pulse profiles (Strickman et al. 1996; Swank 1996), which differ from the more structured profiles typical of other X-ray pulsars (Joss \& Rappaport 1984), may also be indicative of a weaker magnetic field in this object.

Lamb et al. (1996) have presented a scenario wherein the bursts in GRO J1744-28 are of type I, resulting from thermonuclear flashes on the mag-

\footnotetext{
${ }^{1}$ A more detailed theory of accretion torques by Ghosh \& Lamb (1979), which allows for the penetration of the magnetosphere by the inner edge of the accretion disk, yields additional correction factors of the order of unity to Equations (1) - (3); these corrections have no significant effect on our results or conclusions.
} 
netic polar caps of a highly magnetized $\left(B \simeq 1 \times 10^{13} \mathrm{G}\right)$ neutron star. While the viability of this scenario can be tested only through detailed calculations, the inferred weakness of the neutron-star magnetic field in GRO J1744-28 seems to exclude the scenario as a model for this source.

On the basis of the above observational evidence and theoretical arguments, we conclude that the bursts from GRO J1744-28 are of type II and probably result from an instability in the accretion flow that is associated with the weak but non-negligible magnetic field of the underlying neutron star (see Lewin \& Joss [1981] and Lewin et al. [1993] for references). Lewin et al. (1996) have reached the same conclusion, based on a somewhat different set of arguments. Using substantially less observational evidence than is now available, Daumerie et al. (1996) and Kouveliotou et al. (1996b) have also argued that the bursts in GRO J1744-28 result from spasmodic accretion onto a neutron star.

It is interesting to speculate that the X-ray pulsars, GRO J1744-28, the Rapid Burster, and the type I X-ray bursters may form a continuum of possible behaviors of an accreting neutron star, with the neutron-star magnetic moment, $\mu$, serving as the crucial parameter that determines the mode of behavior in any given source. In the case of X-ray pulsars, $\mu$ would be sufficiently large that the accretion flow is funneled onto the magnetic polar caps of the neutron star, producing periodic X-ray pulsations but suppressing the thermonuclear flashes that would give rise to type I X-ray bursts, even if the accretion rate is relatively low. In GRO J1744-28, $\mu$ would still be sufficiently large to funnel the accretion flow but small enough to permit an instability in the flow that gives rise to type II X-ray bursts. In the Rapid Burster, which is the only known source to display both type I and type II bursts (at least on a regular basis), $\mu$ would be too small to funnel the accretion flow, so that the accretion onto the neutron-star surface is roughly isotropic and type I bursts can occur, but still sufficiently large to produce the accretion instability that results in type II bursts. Finally, if $\mu$ is even smaller than in the Rapid Burster, the magnetic field should have relatively little effect upon the accretion flow; as long as the accretion rate is not too high, the source will be a type I X-ray burster. If this picture is correct, an important challenge for both observers and theorists will be to determine the critical values of $\mu$ that distinguish the various modes of behavior of an accreting neutron star.

\section{Current status and evolutionary history of the binary system}

The very small mass function of the GRO J1744-28 system implies either that we are viewing the system almost exactly pole-on (Daumerie et al. 1996), a possibility that has a very small a priori probability, or that the companion star has a very low mass $\left(\leqslant 0.2 M_{\odot}\right)$ (Daumerie et al. 1996; Lamb et al. 1996; Sturner $\&$ Dermer 1996). In the latter case, a viable model for the binary is one in which the mass transfer is driven by the internal evolution of a giant companion that has already lost most of its hydrogen-rich envelope via the mass-transfer process (Daumerie et al. 1996; Lamb et al. 1996). This type of evolutionary scenario (Webbink, Rappaport, \& Savonije 1983) has previously been adopted as a model for the prior evolutionary history of wide-binary radio pulsars with nearly circular orbits (Joss \& Rappaport 1983; Paczyński 1983; Savonije 1983; Joss, Rappaport, \& Lewis 1987; Rappaport et al. 1995). 
By combining the known orbital period and mass function of the GRO J1744-28 system with Kepler's third law, the relation between the orbital separation and the effective radius of the Roche lobe of the secondary (giant) star, the assumption that the Roche lobe is filled during mass transfer, and the core mass - radius relation for low-mass giants (Webbink et al. 1983; Joss et al. 1987; Rappaport et al. 1995), we can derive useful constraints on the masses of the core and residual hydrogen-rich envelope as functions of the orbital inclination, $i$. First, using the measured mass function of GRO J1744-28, we obtain

$$
\left(M_{\mathrm{c}}+M_{\mathrm{env}}\right)^{3} \sin ^{3} i /\left(M_{\mathrm{c}}+M_{\mathrm{env}}+M_{\mathrm{ns}}\right)^{2} \simeq 1.3 \times 10^{-4},
$$

where $M_{\mathrm{c}}$ and $M_{\text {env }}$ are the mass of the hydrogen-exhausted core of the giant and the mass of the surrounding envelope (i.e., the remainder of the star), respectively, in units of solar masses, $M_{\mathrm{g}}=\left(M_{\mathrm{c}}+M_{\mathrm{env}}\right) M_{\odot}$ being the total mass of the giant, and $M_{\mathrm{ns}}$ is the mass of the neutron star. Second, the combination of Kepler's third law with the expression (Kopal 1959) for the effective radius of the Roche lobe of the giant yields

$$
\left(M_{\mathrm{c}}+M_{\text {env }}\right) / R_{\mathrm{g}}^{3} \simeq 1.0 \times 10^{-3},
$$

where $R_{\mathrm{g}}$ is the radius of the giant in units of solar radii. Third, there is a fairly tight relationship between $R_{\mathrm{g}}$ and $M_{\mathrm{c}}$ for low-mass giants; adapting the results of Rappaport et al. (1995) to cases where $M_{\mathrm{c}} \leq 0.25$, we find that this relationship can be well fit by

$$
\log R_{\mathrm{g}} \simeq-0.02617+1.18855 M_{\mathrm{c}}+11.8157 M_{\mathrm{c}}^{2}, M_{\mathrm{c}} \leq 0.25 .
$$

Here, $R_{\mathrm{g}}$ has an uncertainty of a factor of $\sim 1.3$, owing to the unknown metallicity and initial main-sequence mass of the giant and to the uncertainties inherent in the mixing-length theory of convection. We solve Equation (5) numerically, in order to obtain $\left(M_{\mathrm{c}}+M_{\text {env }}\right.$ ) as a function of $i$ for any chosen value of $M_{\mathrm{ns}}$. The result is then substituted into Equation (6) to obtain $R_{\mathrm{g}}$ as a function of $i$. Finally, we substitute into Equation (7) to obtain both $M_{\mathrm{c}}$ and $M_{\text {env }}$ as functions of $i$ alone. The results of this calculation are shown in Figure 1.

The spread in the values of $M_{\mathrm{c}}$ and $M_{\text {env }}$ in Figure 1 reflects the uncertainty in $R_{\mathrm{g}}$ at any given value of $M_{\mathrm{c}}$ and the uncertainty in $M_{\mathrm{ns}}$, which, for the purpose of this calculation, we take to lie in the range from 1.2 to $1.8 M_{\odot}$. As can be seen from the figure, the largest permissible inclination angle is $\sim 20^{\circ}$; for an isotropic distribution of orbital inclinations, the a priori probability for a given system to have $i \lesssim 20^{\circ}$ is $\sim 6 \%$. It is noteworthy that the largest, and hence most probable, inclination angles correspond to values of $M_{\text {env }}$ approaching zero. This suggests the possibility that the GRO J1744-28 system is in the final stages of mass transfer; at the current spin period of the neutron star, the magnetic field strength may be marginally sufficient (Ruderman \& Sutherland 1975), once accretion has ceased, to cause the neutron star to turn on as a wide-binary radio pulsar with a nearly circular orbit.

We have used Equation (7) and the core mass - luminosity relation for low-mass giants (Rappaport et al. 1995) to estimate the observable parameters of the companion star in the GRO J1744-28 system. We obtain a luminosity, 


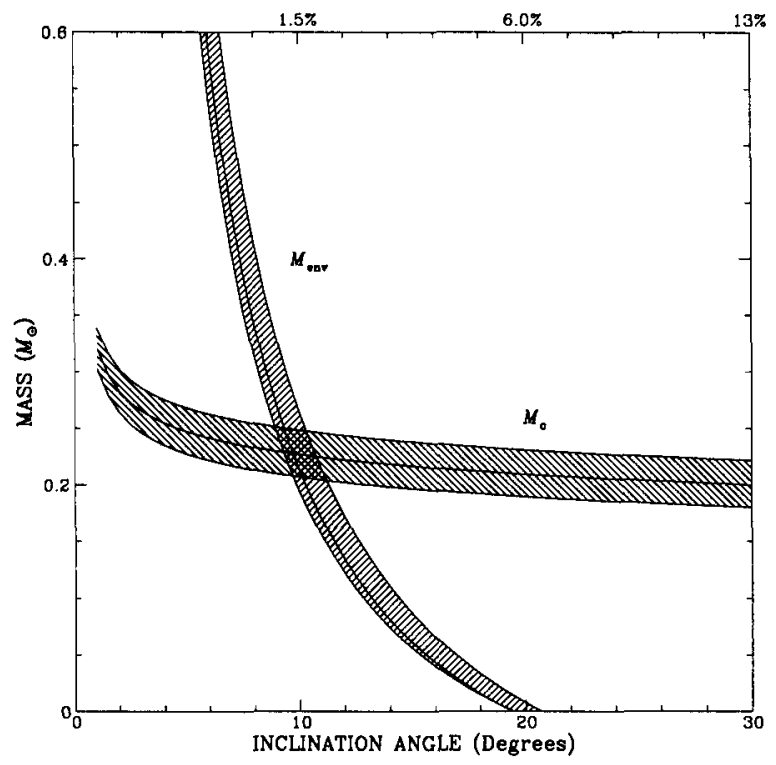

Figure 1. $\quad M_{\mathrm{c}}$ and $M_{\text {env }}$ as functions of $i$. The curve running through the center of each band indicates the most probable mass values, under the assumption that $M_{\mathrm{ns}}=1.4 M_{\odot}$. The scale at the top of the figure shows the $a$ priori probability that a system chosen at random from an ensemble of systems with isotropically oriented inclination angles would have a value of $i$ equal to or less than the indicated value.

effective radius, and effective temperature of $\sim 20 L_{\odot}, \sim 6 R_{\odot}$, and $\sim 5000 \mathrm{~K}$, respectively. These results are insensitive to the assumed values of $M_{\text {env }}$ and $i$. The detection of the optical counterpart to GRO J1744-28 by Kouveliotou (1996) neither confirms nor contradicts these predictions, because of the unknown and possibly large interstellar extinction and reddening in the direction of the source, and because much of the observed optical emission may come from the accretion disk (Kouveliotou 1996).

Starting from the assumptions that the initial mass of the giant was $1 M_{\odot}$ and that the envelope presently contributes a small fraction of the mass of the giant, we have calculated the prior evolutionary history of the binary system. The results of this calculation are shown in Figure 2. We find that the orbital period increases from $\sim 1$ day to $\sim 11.8$ days and $M_{c}$ increases from $\sim 0.15 M_{\odot}$ to $\sim 0.21 M_{\odot}$ during the mass-transfer phase of the binary evolution, which lasts for $\sim 8 \times 10^{8} \mathrm{yr}$. Note that the long-term average mass-transfer rate at the pres- 


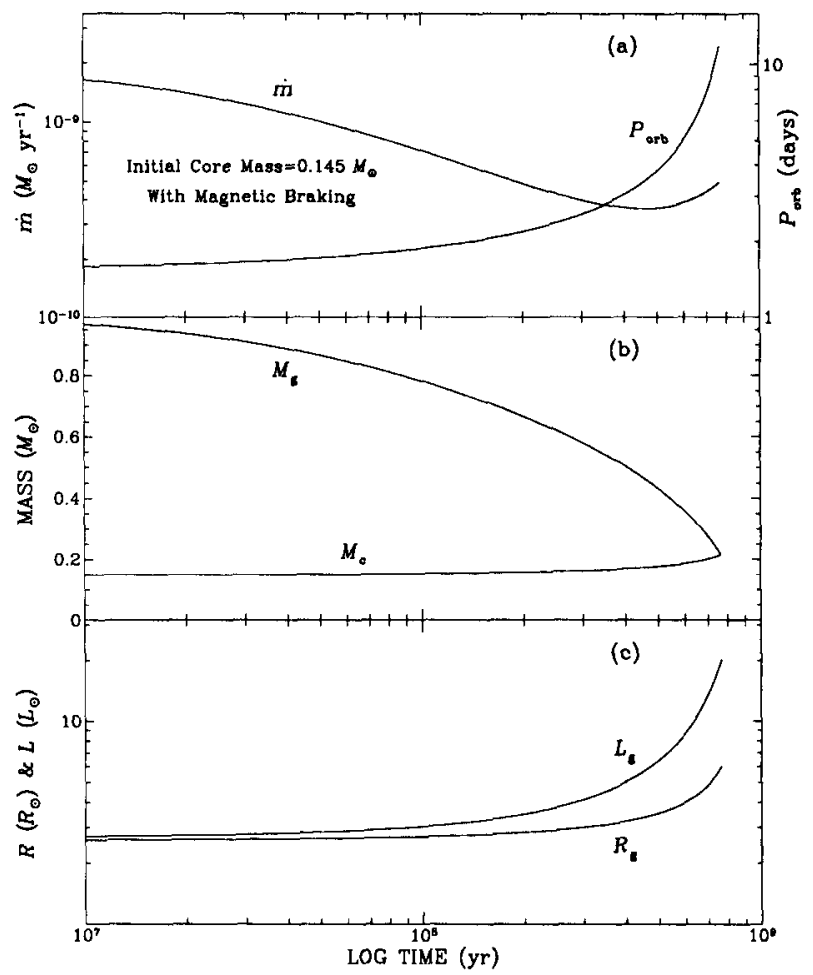

Figure 2. A possible prior evolutionary history for the GRO J1744-28 binary system. At the onset of mass transfer (time $t=0$ ), the masses of the giant donor star, its core, and the neutron star are taken to be $1.0,0.15$, and $1.4 M_{\odot}$, respectively. The effects of both the internal evolution of the giant (see text for references) and magnetic braking with a braking index of 3 (Verbunt \& Zwaan 1981; Rappaport, Verbunt, \& Joss 1983) have been included in the evolutionary calculations. Half of the mass lost by the giant is assumed to be accreted by the neutron star, with the other half lost from the system while carrying the same specific angular momentum as that of the neutron star. Thus, for the purpose of this evolutionary calculation only, the current mass of the neutron star is taken to be $\sim 1.8 M_{\odot}$. Shown as functions of $\log t$ are (a) the accretion rate, $\dot{m}$, onto the neutron star (left-hand scale) and the binary orbital period, $P_{\text {orb }}$ (right-hand scale), (b) the mass of the giant, $M_{\mathrm{g}}$, and that of its core, $M_{\mathrm{c}}$, and (c) the luminosity, $L_{\mathrm{g}}$, and radius, $R_{\mathrm{g}}$, of the giant. 
ent epoch is predicted to be $\sim 3 \times 10^{-10} M_{\odot} \mathrm{yr}^{-1}$, corresponding to a long-term average X-ray luminosity of $\sim 4 \times 10^{36} \mathrm{ergs} \mathrm{s}^{-1}$. From Equation (2), we then find that the equilibrium rotation rate of the neutron star (i.e., the rotation rate which equals the long-term average orbital frequency at the inner edge of the accretion disk) is $\sim 2.5 \mathrm{~Hz}$. This value is remarkably close to the observed $2.1 \mathrm{~Hz}$ pulse rate of GRO J1744-28, supporting our estimate for $B$ in Equation (4).

Three possibilities present themselves as mechanisms for producing the current transient outburst of the GRO J1744-28 system. The first, and perhaps least interesting, possibility is that the outburst is the result of an accretion-disk instability (Cheng and Lin 1992; Cannizzo 1993; Bildsten and Brown 1996; and references in these works). The remaining two possibilities are both related to the evolutionary status of the binary system. If, as suggested above, the system is in the terminal stages of mass transfer, then the current outburst may be the result of a final hydrogen-burning flash that occurred as the residual hydrogenrich envelope collapsed onto the core of the giant (Härm \& Schwarzschild 1975). Alternatively, if the envelope has not yet collapsed but its mass has dropped below $\sim 10^{-3} M_{\odot}$, an estimate based on the mixing-length theory of convection (Böhm-Vitense 1958; Cox \& Giuli 1968) indicates that convective heat transport will have become inefficient through a large portion of the envelope. Under these conditions, the velocities of the turbulent convective eddies will become transonic, and there will be relative density fluctuations of the order of unity on effective equipotential surfaces (Cox \& Giuli 1968), including the surface of the Roche lobe. It is then possible that the current transient outburst is a result of the penetration of the Roche lobe by a particularly large density excursion. In this scenario, the duration of the outburst corresponds to the amount of time required for all of the excess matter excreted through the Roche lobe to make its way to the surface of the neutron star, and future outbursts of varying intensity may be expected from this system in the future.

Acknowledgments. We thank W. H. G. Lewin and L. Nelson for helpful discussions, and $\mathbf{J}$. Kiger for assistance in the numerical computations and in the preparation of the figures. Supported in part by NASA grant NAG5-2632.

\section{References}

Alpar, M. A., \& Shaham, J. 1985, Nature, 316, 239

Ayasli, S., \& Joss, P. C. 1982, ApJ, 256, 637

Bildsten, L., \& Brown, E. F. 1996, ApJ, in press

Böhm-Vitense, E. 1958, ZAp, 46, 108

Briggs, M. S. et al. 1996, IAU Circ., No. 6290

Cannizzo, J. K. 1993, ApJ, 419, 318

Cheng, F. H., \& Lin, D. N. C. 1992, ApJ, 389, 714

Cox, J. P., \& Giuli, R. T. 1968, Principles of Stellar Structure and Evolution, Vol. 1 (New York: Gordon \& Breach)

Daumerie, P., Kalogera, V., Lamb, F. K., \& Psaltis, D. 1996, Nature, 382, 141

Finger, M. H., Koh, D. T., Nelson, R. W., Prince, T. A., Vaughan, B. A., \& Wilson, R. B. 1996a, IAU Circ., No. 6285

Finger, M. H., Wilson, R. B., \& van Paradijs, J. 1996b, IAU Circ., No. 6286 
Fishman, G. J. et al. 1995, IAU Circ., No. 6272

Fishman, G. J. et al. 1996, IAU Circ., No. 6290

Ghosh, P., \& Lamb, F. K. 1979, ApJ, 234, 296

Giles, B., \& Strohmayer, T. 1996, IAU Circ., No. 6338

Härm, R., \& Schwarzschild, S. 1975, 200, 324

Joss, P. C. 1977, Nature, 230, 310

Joss, P. C. 1978, ApJ, 238, L123

Joss, P. C., \& Li, F. K. 1980, ApJ, 238, 287

Joss, P. C., \& Rappaport, S. 1976, Nature, 264, 219

Joss, P. C., \& Rappaport, S. 1983, Nature, 304, 419

Joss, P. C., \& Rappaport, S. A. 1984, ARA\&A, 22, 537

Kopal, Z. 1959, Close Binary Systems (London: Chapman \& Hall).

Joss, P. C., Rappaport, S., \& Lewis, W. 1987, ApJ, 319, 180

Kommers, J., \& Fox, D. 1996, unpublished

Kouveliotou, C. 1996, IAU Circ., No. 6369

Kouveliotou, C. et al. 1996a, IAU Circ., No. 6286

Kouveliotou, C. et al. 1996b, Nature, 379, 799

Lamb, D. Q., Miller, M. C., \& Taam, R. E. 1996, preprint

Lewin, W. H. G. et al. 1976, ApJ, 207, L95

Lewin, W. H. G., \& Joss, P. C. 1981, Space Sci.Rev., 28, 3

Lewin, W. H. G., Rutledge, R. E., Kommers, J. M., van Paradijs, J., \& Kouveliotou, C. 1996, ApJ, 462, L39

Lewin, W. H. G., van Paradijs, J., \& Taam, R. E. 1993, Space Sci.Rev., 62, 223

Morgan, E. 1996, private communication

Paciesas, W. S., Harmon, B. A., Fishman, G. J., Zhang, S. N., \& Robinson, C. R. 1996, IAU Circ., No. 6284

Paczyński, B. 1983, Nature, 304, 421

Rappaport, S., \& Joss, P. C. 1977, Nature, 266, 683

Rappaport, S., Podsiadlowski, Ph., Joss, P. C., Di Stefano, R., \& Han, Z. 1995, MNRAS, 273, 731

Rappaport, S., Verbunt, F., \& Joss, P. C. 1983, ApJ, 275, 713

Ruderman, M. A., \& Sutherland, P. G. 1975, ApJ, 196, 51

Savonije, G. J. 1983, Nature, 304, 422

Strickman, M. S. et al. 1996, preprint

Sturner, S. J., \& Dermer, C. D. 1996, ApJ, 465, L31

Swank, J. 1996, IAU Circ., No. 6291

Tawara, Y., Hayakawa, S., Kunieda, H., Makino, F., \& Nagase, F. 1982, Nature, 299,38

Verbunt, F., \& Zwaan, C. 1981, A\&A, 100, L7

Webbink, R. F., Rappaport, S., \& Savonije, G. J. 1983, ApJ, 270, 678

Zhang, W. et al. 1996, IAU Circ., No. 6300 


\section{Discussion}

R. Sunyaev: Observations of GRO J1744-28 with GRANAT/WATCH (Sazonov and Sunyaev, IAU Circular No. 6291, 1996) and MIR-KVANT showed that the persistent luminosity of the source exceeded up to 5 times the Eddington limit for a neutron star at the distance of the galactic center. During bursts, the luminosity was another order of magnitude higher for intervals of 5-10 seconds. This time scale exceeds any of the important dynamical times relevant to the release of energy driven by accretion onto a neutron star with $B<10^{11}$ gauss. It seems clear that only accretion onto an object with a stronger magnetic field (which would lower the radiative opacity) and a very high accretion column could emit such a highly super-Eddington luminosity. (Basko and Sunyaev [MNRAS, 1976] showed that an accretion column becomes unstable when the pressure at the base of the column [i.e., the weight of the column per unit surface area] exceeds the value of $B^{2} /(8 * \pi)$ at the surface of the neutron star.) I think that any model for GRO J1744-28 must explain how this source achieves superEddington luminosities, even if the source distance is only 1-2 kpc.

P. Joss: IAU Circular No. 6291 quotes a persistent luminosity of approximately $4 \times 10^{38} \mathrm{ergs} \mathrm{s}^{-1}$, which is close to the value that we have estimated on the basis of independent arguments. As noted in the circular, moderately superEddington luminosities have previously been detected in SMC X-1 and several other X-ray pulsars. I was, however, unaware of the apparent existence of highly super-Eddington luminosities during bursts from GRO 1744-28. I agree that this observation presents a serious challenge for all existing theories of the nature of the $\mathrm{X}$-ray emission from this source. 